\title{
Spatial distribution and nematic ordering of anisotropic nanoparticles in lamellae and hexagonal phases of block copolymers
}

\author{
Mikhail A. Osipov ${ }^{1,2}$ and Maxim V. Gorkunov ${ }^{3}$ \\ 1 Department of Mathematics, University of Strathclyde, Glasgow G1 1XH, Scotland, UK \\ 2 Topchiev Institute of Petrochemical Synthesis, Russian Academy of Sciences, 119991 Moscow, Russia \\ 3 Shubnikov Institute of Crystallography of Federal Scientific Research Centre "Crystallography and Photonics" of Russian \\ Academy of Sciences, 119333 Moscow, Russia
}

Received: date / Revised version: date

\begin{abstract}
Orientational and translational ordering of anisotropic nanoparticles in the lamellae and hexagonal phases of diblock copolymers have been considered theoretically in the case of strong segregation taking into account the anisotropic interaction between the nanoparticles and the monomers in different blocks. It has been shown that anisotropic nanoparticles are orientationally ordered in the boundary region between the blocks and the nematic order parameter possesses opposite signs in different blocks: the nanoparticles align parallel to the boundary in one block and perpendicular to it in the other. In the hexagonal phase, a weak biaxial ordering of nanoparticles is also induced in the boundary region. Explicit analytical results have been obtained for the distribution of nanoparticles in the lamellae phase. The results are compared with the existing experimental data.
\end{abstract}

\section{Introduction}

Composite polymer nanomaterials that contain metal, oxide, silicate or semiconductor nanoparticles (NPs) have a number of advantages over pure polymer systems. It has been shown that the introduction of NPs improves the mechanical, thermal, electric and dielectric characteristics of polymer materials [1-7]. Microphase separation in block copolymers can stabilize the spatially inhomogeneous distribution of the NPs, which in principle enables one to develop highly ordered hybrid materials with controlled parameters. Such materials can be used as novel membranes and employed in nanophotonics $[8,9]$.

Recently, NPs have been selectively confined in one block domain and assembled into quantum dots, nanowires, and nanoring structures [10-13] by varying the composition of copolymers and their chemical structure. In contrast to nanostructures produced by the lithography techniques, such systems are self-organizing and essentially three dimensional. The assembly of spherical NPs in block copolymers has been of significant interest since the theoretical and experimental studies undertaken during the past decade $[10,11,14,15]$. At the same time, recent studies of nanorods (NRs) in homopolymer [16-19] show that dispersion and alignment of anisotropic NPs can result in a tunable structure-property relationship.

With few exceptions (see, for example, Refs. [20-23]) the existing theoretical studies of polymer nanocomposites are confined to the case of isotropic NPs [24]. So far, there has been no systematic investigation of the effect of NP anisotropy on the phase behavior of diblock copolymers and, reciprocally, of the effect of microphase separation on the orientational and translational ordering of anisotropic NPs. At the same time, the NP anisotropy is known to play a significant role in the related soft matter systems. In particular, it has been shown that anisotropic composites based on low molecular weight liquid crystals (LCs) doped with NPs possess a number of improved characteristics in comparison with conventional LCs including lower threshold voltages and switching times of LC displays [2529]. The introduction of NPs in various LC materials enables one to extend the range of several LC phases, to raise the temperature of phase transitions and to improve the conditions of lasing in the LC matrix doped with dyes [30-34]. Recently, a molecular theory has been developed to explain the effect of NPs on thermodynamic and dielectric properties of low molecular weight LCs [35-39]. One expects that composites based on LC polymers and anisotropic NPs will offer a number of benefits over the conventional LC polymers.

Quite generally, the ability of block copolymers to form organized structures with a period in the range of tens to hundreds of nanometers, can be widely used in the development of nanomaterials for lithography, membrane technology, light transmission, data recording, etc. However, one of the serious unsolved technological problems here is the aligning of anisotropic phases, mainly the hexagonal and the lamellae ones, with respect to the substrates. In- 
duced orientational order of strongly anisotropic NPs in block copolymers may, in principle, be employed to align these phases by external electric field.

There exists an experimental evidence that anisotropic NPs may be aligned in lamellae and hexagonal phases of block copolymers even at very low concentrations. For example, it has been found that poly(ethylene glycol) (PEG) functionalized gold nanorods are aligned parallel to the lamellae planes [40]. At the same time, the polystyrene functionalized NRs are orientationally ordered in the hexagonal phase of the same block copolymer with the long axes perpendicular to the cylinders $[41,42]$. In contrast, the alkyl phosphonic acid capped NRs are ordered parallel to the cylinders in a different block copolymer [43].

Therefore, the effect of NPs on the properties of block copolymers is twofold. On the one hand, NPs may affect the relative stability of different phases and shift the corresponding transition temperatures. On the other hand, the microphase separation in the polymer matrix may induce inhomogeneous periodic distribution of NPs. This, in turn, may affect dielectric, optical and mechanical properties of block copolymers. In this paper we address the second topic, and calculate the spatial distribution of anisotropic NPs in lamellae and hexagonal phases as well as the orientational order parameter profiles in these phases, taking into account both isotropic and anisotropic interactions between NPs and monomers. As far as we know, the anisotropic interaction between NPs and polymer chains has not been taken into account in the existing theory of polymer nanocomposites.

The most interesting effects are predicted in the vicinity of the interface between the blocks. In this region, anisotropic NPs may interact simultaneously with different monomers located in adjacent blocks. This asymmetric interaction generally induces nematic orientational order of anisotropic NPs in the interfacial region. Moreover, NPs may align parallel and perpendicular to the interface on different sides of the boundary. In the hexagonal phase, the curvature of the cylindrical interface also induces the biaxial order of NPs.

The paper is arranged as follows: in Sections 2 and 3 the NP density and nematic order parameter profiles are calculated in the lamellae phases with strong and weak block segregation respectively. The case of the hexagonal phase with strong segregation is considered in Section 4. Section 5 contains a discussion.

\section{Lamellae phase. Strong segregation}

Let us consider a small admixture of anisotropic uniaxial NPs in the idealized diblock-copolymer with fixed density distribution of A and B segments. In the simplest lamellae phase, the total periodicity is $d=d_{A}+d_{B}$, where $d_{A}$ is the thickness of the A-rich layer and $d_{B}$ is that of the B-rich layer, respectively.

Let us first consider the ideal copolymer in the strong segregation limit. In this case, the block $\mathrm{A}$ is composed only of monomers A while the block B is composed of monomers B. Denoting the densities of the monomers A

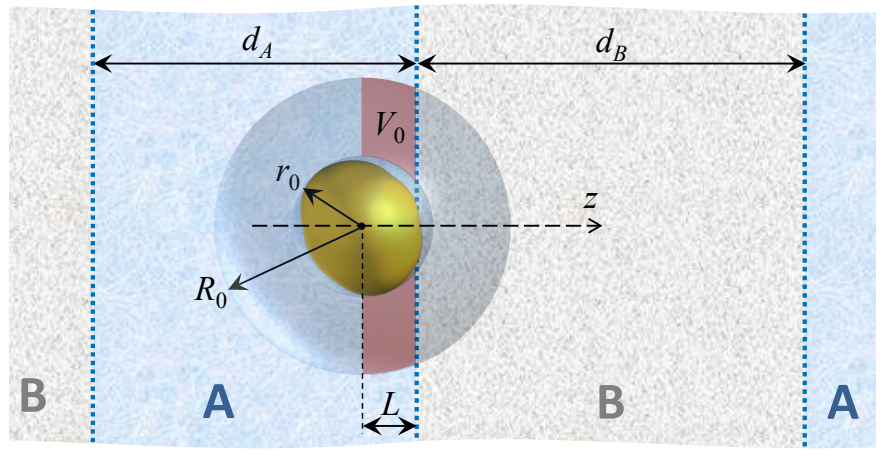

Fig. 1. NP embedded in a lamellae diblock copolymer with strong segregation.

and $\mathrm{B}$ in the corresponding pure polymers as $\rho_{0 A}$ and $\rho_{0 B}$ respectively, one can express the average number density of monomers $\mathrm{A}$ as $\rho_{A}(\mathbf{r})=\rho_{0 A}$ when $0<z<d_{A}, \rho_{A}(\mathbf{r})=$ 0 when $d_{A}<z<d$, and $\rho_{A}(z)=\rho_{A}(z+d)$ for all $z$ as it is a periodic function with the period $d$. The average number density of monomers $\mathrm{B}$ is given by similar expressions: $\rho_{B}(\mathbf{r})=\rho_{0 B}$ when $d_{A}<z<d, \rho_{B}(\mathbf{r})=0$ when $0<z<$ $d_{A}$ and $\rho_{B}(z)=\rho_{B}(z+d)$ for all $z$.

In this paper we use a simple expression for the interaction potential between the $i$-th NP and the monomers, which is composed of the isotropic and anisotropic parts:

$$
\begin{aligned}
U_{i}=\sum_{l=l_{A}}\left[J_{A}\left(r_{i l}\right)+I_{A}\left(r_{i l}\right) P_{2}\left(\mathbf{a}_{i} \cdot \mathbf{u}_{i l}\right)\right]+ \\
\sum_{l=l_{B}}\left[J_{B}\left(r_{i l}\right)+I_{B}\left(r_{i l}\right) P_{2}\left(\mathbf{a}_{i} \cdot \mathbf{u}_{i l}\right)\right]
\end{aligned}
$$

where $\mathbf{r}_{i l}=\mathbf{r}_{i}-\mathbf{r}_{l}$, and $\mathbf{u}_{i l}$ is the unit vector in the direction of $\mathbf{r}_{i l} ; \mathbf{r}_{i}$ is the position vector of the $i$-th NP and $\mathbf{a}_{i}$ is the unit vector in the direction of its long axis; $\mathbf{r}_{l}$ is the position vector of the corresponding monomer (A or B); the functions $J_{A}\left(r_{i l}\right), I_{A}\left(r_{i l}\right)$ and $J_{B}\left(r_{i l}\right), I_{B}\left(r_{i l}\right)$ describe the isotropic and anisotropic coupling between NPs and monomers $\mathrm{A}$ and $\mathrm{B}$; and $P_{2}(x)$ is the second Legendre polynomial.

The anisotropic interaction between isotropic monomers and anisotropic NPs in Eq. (1) effectively couples the long NP axis $\mathbf{a}_{i}$ and the unit vector $\mathbf{u}_{i l}$ pointing from the particle to the monomer. The anisotropic part of the potential vanishes after integration over the unit intermolecular vector $\mathbf{u}_{i j}$. One notes that the anisotropic interaction of this kind has not been taken into consideration in the existing theory of polymer nanocomposites [24]. At the same time, such an anisotropic interaction potential appears naturally in the general expression for the dipole-dipole dispersion intermolecular interaction $[44,45]$ and describes a coupling between the NP anisotropic polarizability and isotropic polarizability of a monomer. This potential has also been used in the molecular theory of Smectic A - Smectic C phase transition $[45,46]$. In contrast, the Maier-Saupe type interaction potential, which promotes the nematic ordering in LCs [45], is proportional to the anisotropy of the polarizabilities of both interacting molecules0. One notes also that in this model we do take into account the geo- 
metrical anisotropy of a NP explicitly but consider only the anisotropy of the corresponding interaction potential which is determined by by the anisotropic polarizability of a NP.

In the molecular field approximation, the single NP spatial and orientational distribution function is given by the Boltzman expression:

$$
f\left(\mathbf{a}_{i}, \mathbf{r}_{i}\right)=Z^{-1} \exp \left[-U_{M F}\left(\mathbf{a}_{i}, \mathbf{r}_{i}\right) / k_{B} T\right],
$$

where $Z$ is the normalization factor and the mean-field potential $U_{M F}\left(\mathbf{a}_{i}, \mathbf{r}_{i}\right)$ is expressed as:

$$
\begin{aligned}
& U_{M F}\left(\mathbf{a}_{i}, \mathbf{r}_{i}\right)= \\
& \qquad\left[J_{A}\left(r_{i l}\right)+I_{A}\left(r_{i l}\right) P_{2}\left(\mathbf{a}_{i} \cdot \mathbf{u}_{i l}\right)\right] \rho_{A}\left(\mathbf{r}_{l}\right) d^{3} \mathbf{r}_{l}+ \\
& \quad \int\left[J_{B}\left(r_{i l}\right)+I_{B}\left(r_{i l}\right) P_{2}\left(\mathbf{a}_{i} \cdot \mathbf{u}_{i l}\right)\right] \rho_{B}\left(\mathbf{r}_{l}\right) d^{3} \mathbf{r}_{l} .
\end{aligned}
$$

The local scalar NP nematic order parameter with respect to the block interface normal $\mathbf{k}$ is then expressed as

$$
S(\mathbf{r})=\left\langle P_{2}\left(\mathbf{a}_{i} \cdot \mathbf{k}\right)\right\rangle=\frac{\int P_{2}\left(\mathbf{a}_{i} \cdot \mathbf{k}\right) f\left(\mathbf{a}_{i}, \mathbf{r}\right) d^{2} \mathbf{a}_{i}}{\int f\left(\mathbf{a}_{i}, \mathbf{r}\right) d^{2} \mathbf{a}_{i}},
$$

while the average density distribution of NPs is given by

$$
\rho(\mathbf{r})=\rho_{N} \int f\left(\mathbf{a}_{i}, \mathbf{r}\right) d^{2} \mathbf{a}_{i}
$$

where $\rho_{N}$ is the average NP number density .

The NP distribution can be determined only within a particular model of the NP-monomer interaction. Let us assume for simplicity that $J_{\alpha}(r)=J_{\alpha} r^{-6}$ and $I_{\alpha}(r)=$ $I_{\alpha} r^{-6}$ (which corresponds, e.g., to the dispersion intermolecular interaction), where $\alpha=A, B$, and there is a cut-off at small $r=r_{0}$, where $r_{0}$ is the NP radius. At larger $r$, there is another cut-off at $r=R_{0}$, where $R_{0}$ is the range of interaction, which is assumed to be relatively small, e.g., $R_{0}<d_{A} / 2, d_{B} / 2$.

In this case, some of the integrals in Eq. (3) can be taken analytically. Let us consider the mean-field potential $U_{M F}(\mathbf{a}, \mathbf{r})$ acting on NP at some point $\mathbf{r}$ (see Fig. 1). Taking into account that the interaction range is smaller than the half-widths of the blocks, one concludes that NP at point $\mathbf{r}$ interacts with the monomers inside the same block and with the monomers of the other type in the closest adjacent block, if NP is sufficiently close to the boundary between the blocks. We denote by $L$ the distance from the NP center to the closest boundary between the blocks.

Taking into account that the interaction potentials (1) are even functions of $\mathbf{r}_{i l}$, one can express the mean-field potential (3) acting on a particle in the block A as:

$$
\begin{gathered}
U_{M F}=\frac{1}{2} \int_{r_{0}<r<R_{0}} d V \times \\
{\left[\left(\rho_{0 A} J_{A}+\rho_{0 B} J_{B}\right)+\left(\rho_{0 A} I_{A}+\rho_{0 B} I_{B}\right) P_{2}(\mathbf{a} \cdot \mathbf{u})\right] r^{-6}-} \\
\int_{V_{0}} d V\left[\Delta J+\Delta I P_{2}(\mathbf{a} \cdot \mathbf{u})\right] r^{-6} .
\end{gathered}
$$
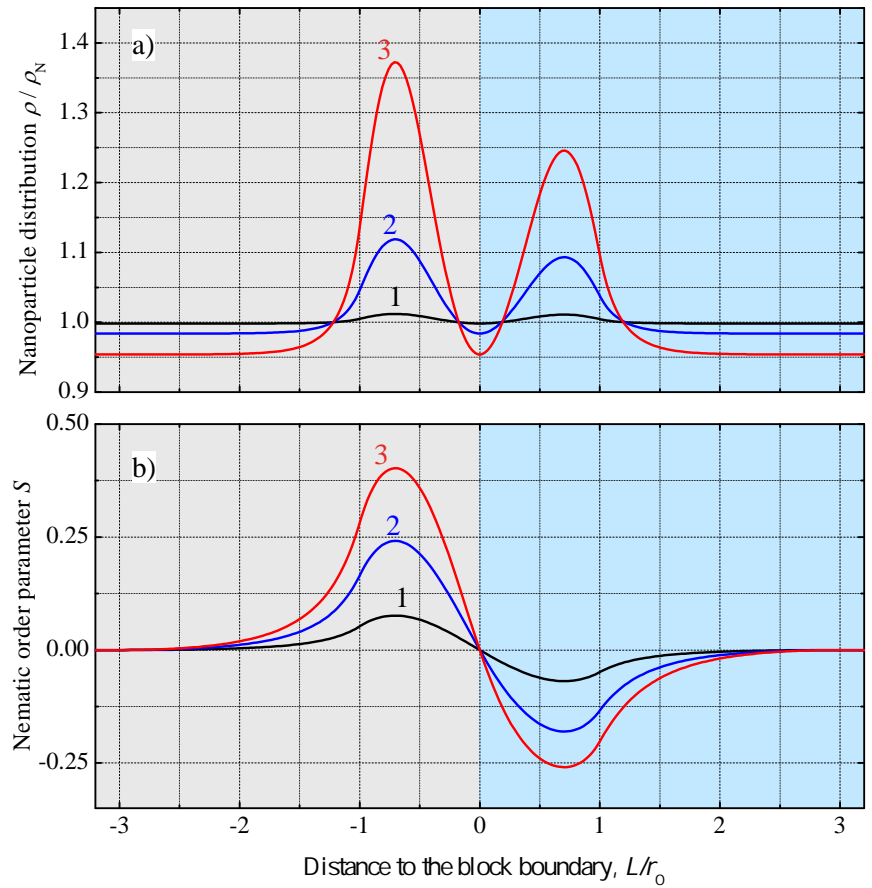

Fig. 2. NP local density (a) and nematic order parameter (b) inside a lamellae diblock copolymer with strong segregation. The block thickness is $d_{A}=d_{B}=10 r_{0}$, the interaction radius $R_{0}=3 r_{0}$, the isotropic interaction constant $\Delta J=0$ and the anisotropic constant $\Delta I=k_{B} T, 3 k_{B} T, 5 k_{B} T$ for the lines from 1 to 3 respectively.
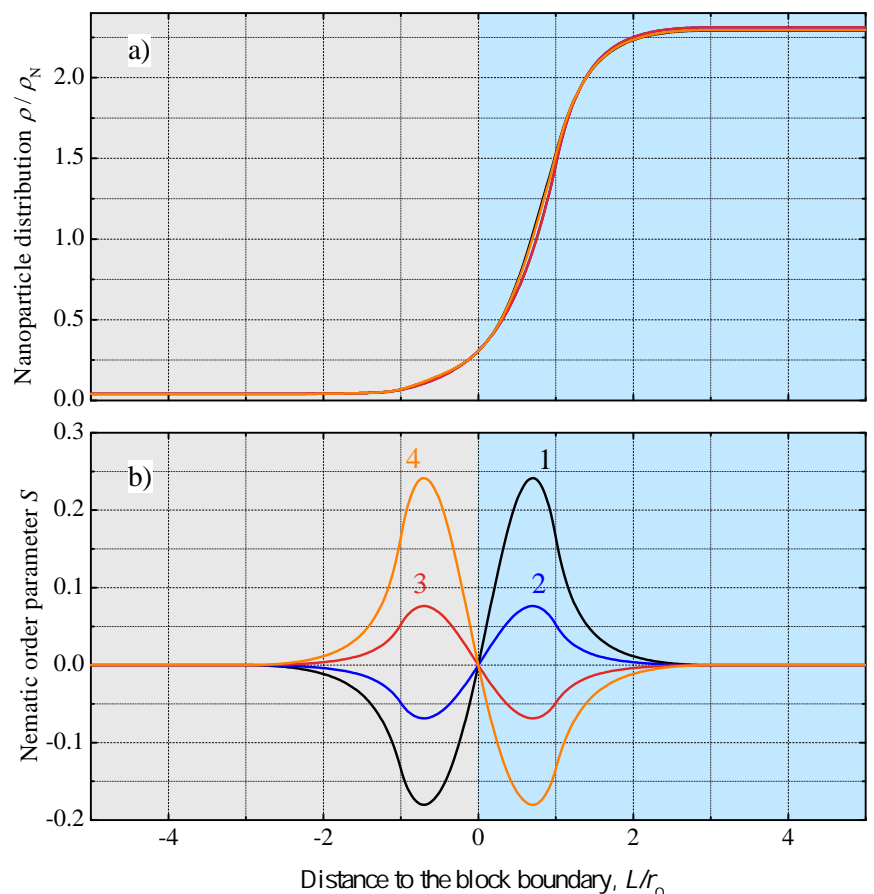

Fig. 3. The same as in Fig. 2 for the isotropic interaction constant $\Delta J=k_{B} T$ and the anisotropic constant $\Delta I=$ $-3 k_{B} T,-k_{B} T, k_{B} T, 3 k_{B} T$ for the lines from 1 to 4 respectively. 
where we have identified a half of the integral over the spherical volume $r_{0}<r<R_{0}$ with the integral over a half of this volume. For a particle centered in block B, one has to reverse the sign of the last term. Here the differences of the interaction parameters are introduced as $\Delta J=\rho_{0 B} J_{B}-\rho_{0 A} J_{A}$, and $\Delta I=\rho_{0 B} I_{B}-\rho_{0 A} I_{A}$. The first integral is taken over the volume of the whole interaction sphere while the second one is taken over its fragment $V_{0}$ confined between the plane of the particle and the block boundary (highlighted in red in Fig. 1). The first term in Eq. (6) yields a constant contribution which is independent of a and $L$, and therefore it can be neglected. The rest of the mean-field potential can be evaluated in a spherical coordinate system with the polar $z$-axis along the normal to the block boundary $\mathbf{k}$, the azimuthal angle $\phi$, and the polar angle $\theta$ measured from the $z$ axis. Then $\mathbf{u}=\mathbf{k} \cos \theta+\mathbf{x} \sin \theta \cos \phi+\mathbf{y} \sin \theta \sin \phi$.

For $L>R_{0}$ one obtains the following expression for the mean-field potential:

$$
\begin{gathered}
U_{M F}^{(0)}(\mathbf{a}, L)=-\int_{r_{0}}^{R_{0}} r^{-4} d r \int_{0}^{1} d \cos \theta \int_{0}^{2 \pi} d \phi \times \\
{\left[\Delta J+\Delta I P_{2}(\mathbf{a} \cdot \mathbf{u})\right]=} \\
=\frac{2 \pi}{3} \Delta J\left(R_{0}^{-3}-r_{0}^{-3}\right) .
\end{gathered}
$$

For an intermediate $r_{0}<L<R_{0}$, the potential can be expressed as

$$
\begin{gathered}
U_{M F}^{(1)}(\mathbf{a}, L)= \\
-\left[\int_{r_{0}}^{L} d r \int_{0}^{1} d \cos \theta+\int_{L}^{R_{0}} d r \int_{0}^{L / r} d \cos \theta\right] \times \\
2 \pi r^{-4}\left[\Delta J+\Delta I P_{2}(\cos \theta) P_{2}((\mathbf{k} \cdot \mathbf{a}))\right]= \\
=\frac{2 \pi}{3} \Delta J\left(L^{-3}-r_{0}^{-3}\right)+ \\
+\frac{\pi L}{2}\left[\Delta J-\frac{\Delta I}{2} P_{2}(\mathbf{k} \cdot \mathbf{a})\right]\left(R_{0}^{-4}-L^{-4}\right)+ \\
\frac{\pi L^{3}}{6} \Delta I P_{2}(\mathbf{k} \cdot \mathbf{a})\left(R_{0}^{-6}-L^{-6}\right),
\end{gathered}
$$

For a particle very close to the block boundary, $L<r_{0}$, one obtains:

$$
\begin{gathered}
U_{M F}^{(2)}(\mathbf{a}, L)=-\int_{r_{0}}^{R_{0}} d r \int_{0}^{L / r} d \cos \theta \times \\
2 \pi r^{-4}\left[\Delta J+\Delta I P_{2}(\cos \theta) P_{2}((\mathbf{k} \cdot \mathbf{a}))\right]= \\
=\frac{\pi L}{2}\left[\Delta J-\frac{\Delta I}{2} P_{2}(\mathbf{k} \cdot \mathbf{a})\right]\left(R_{0}^{-4}-r_{0}^{-4}\right)+ \\
\frac{\pi L^{3}}{6} \Delta I P_{2}(\mathbf{k} \cdot \mathbf{a})\left(R_{0}^{-6}-r_{0}^{-6}\right) .
\end{gathered}
$$

One can verify that the obtained expressions (7), (8) and (9) are continuous at $L=r_{0}$ and $L=R_{0}$. The expressions can easily be generalized to the case when NP is located inside the block $\mathrm{B}$. In this case, the distance $L$ is
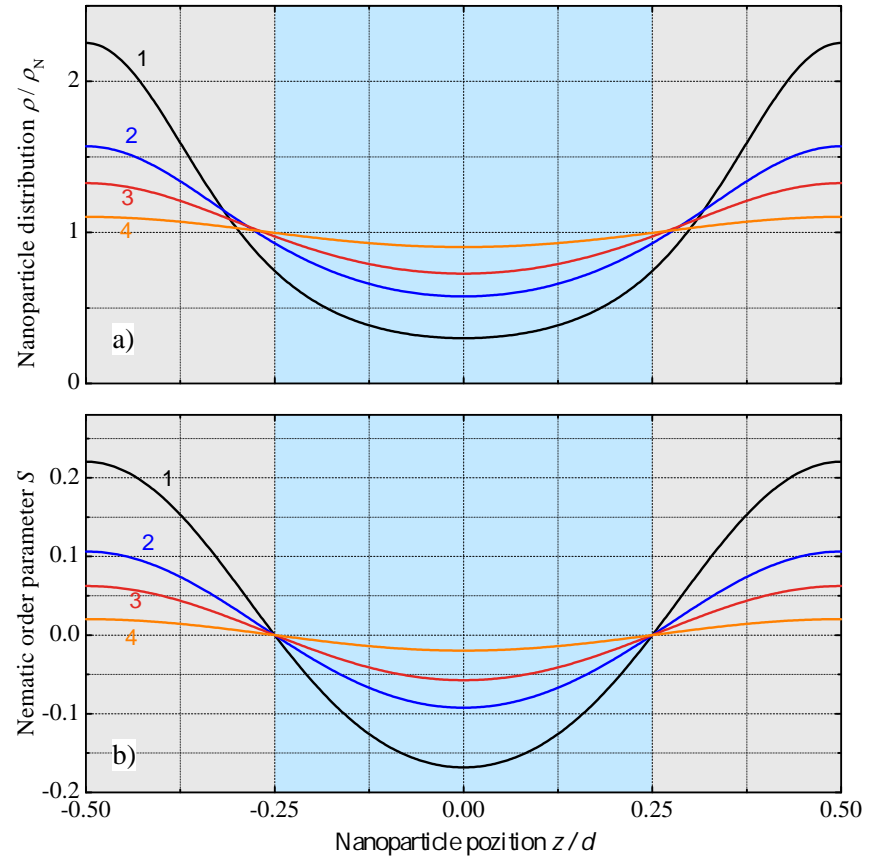

Fig. 4. NP local density and nematic order parameter inside a lamellae diblock copolymer with weak segregation. The coordinate $z$ is normalized by the structure period $d$, the isotropic interaction constant is $\Delta J_{q}=k_{B} T$ and the anisotropic constant is $\Delta I_{q}=k_{B} T$, and the density spatial modulation is varied as $\delta=1,0.5,0.3,0.1$ for the lines from 1 to 4 respectively.

negative. Note that when the particle is located close to the block boundary, the potential (9) is an odd function of $L$ and vanishes when the particle center is directly at the boundary independently of the particle orientation.

Thus we have obtained the analytical expression for the NP distribution in the lamellae phase of the diblock copolymer. The orientational order parameter profile can now be obtained by a numerical integration in Eq. (4). Representative NP density and nematic order parameter profiles are shown in Figs. 2 and 3.

One can readily see in Fig. 2 that in the case $\Delta J=0$ and $\Delta I \neq 0$ (that is when the effective isotropic interaction of NP with monomers A and B is the same while the corresponding anisotropic interaction constants are different) the concentration of NPs is nearly the same throughout the blocks A and B except for the boundary region, where the concentration may be strongly affected by the anisotropic interactions. In this case, the substantial amount of NPs is concentrated in the boundary region, and the concentration is different in the blocks $\mathrm{A}$ and $\mathrm{B}$ close to the boundary. The latter is, in principle, natural as the interface is not a mirror plane.

In contrast, even for relatively small values of $\Delta J$, the NP density profile is only weakly dependent on the strength of the anisotropic interaction $\Delta I$ (see Fig. 3), and NPs are predominantly located in the block A which is characterized by the strongest interaction between NPs and monomers. Similarly to the previous case, NPs are orientationally ordered only in the interfacial region on both 
sides of the boundary. As seen in Fig. 3, the long NP axes are aligned parallel and perpendicular to the block boundary on its different sides, and the nematic order parameter profiles are nearly antisymmetric.

One notes that on the microscopic scale anisotropic NPs may be aligned parallel or perpendicular to the polymer chains. However, far away from the boundaries there is no macroscopic orientational order of NPs because each polymer block is macroscopically isotropic in the bulk.

\section{Lamellae phase. Weak segregation}

At any point in an incompressible copolymer, the local densities $\rho_{A}(\mathbf{r})$ and $\rho_{B}(\mathbf{r})$ satisfy the following equation (see e.g. [47]):

$$
\frac{\rho_{A}(\mathbf{r})}{\rho_{0 A}}+\frac{\rho_{B}(\mathbf{r})}{\rho_{0 B}}=1 .
$$

Weak segregation can be described by a single spatial harmonic of the density modulation and then the distributions of the monomers $\mathrm{A}$ and $\mathrm{B}$ are given by:

$$
\rho_{A}(\mathbf{r})=\rho_{0 A}[1-\delta \cos (\mathbf{q} \cdot \mathbf{r})], \rho_{B}(\mathbf{r})=\rho_{0 B} \delta \cos (\mathbf{q} \cdot \mathbf{r}),
$$

where the modulation wave vector is $\mathbf{q}=2 \pi \mathbf{k} / d$.

Substituting Eq. (11) into the mean-field potential (3), and using the identity $\cos \left(\mathbf{q} \cdot \mathbf{r}_{l}\right)=\cos \left(\mathbf{q} \cdot \mathbf{r}_{i l}\right) \cos \left(\mathbf{q} \cdot \mathbf{r}_{i}\right)+$ $\sin \left(\mathbf{q} \cdot \mathbf{r}_{i l}\right) \sin \left(\mathbf{q} \cdot \mathbf{r}_{i}\right)$ one obtains:

$$
\begin{aligned}
U_{M F}\left(\mathbf{a}_{i}, \mathbf{r}_{i}\right)= & \delta \cos \left(\mathbf{q} \cdot \mathbf{r}_{i}\right) \int d V \times \\
& {\left[\Delta J(r)+\Delta I(r) P_{2}\left(\mathbf{a}_{i} \cdot \mathbf{u}\right)\right] \cos (\mathbf{q} \cdot \mathbf{r}), }
\end{aligned}
$$

where a constant contribution is neglected. Introducing the interaction parameters

$$
\begin{gathered}
\Delta J_{q}=2 \pi \int r^{2} d r \int d \cos \theta \Delta J(r) \cos (q r \cos \theta), \\
\Delta I_{q}=2 \pi \int r^{2} d r \Delta I(r) \int d \cos \theta P_{2}(\cos \theta) \cos (q r \cos \theta),
\end{gathered}
$$

one can write

$$
U_{M F}\left(\mathbf{a}_{i}, \mathbf{r}_{i}\right)=\delta \cos \left(\mathbf{q} \cdot \mathbf{r}_{i}\right)\left[\Delta J_{q}+\Delta I_{q} P_{2}\left(\mathbf{a}_{i} \cdot \mathbf{k}\right)\right],
$$

where the parameters (13) and (14) can be evaluated numerically for a given model interaction potential. Representative profiles of the NP spatial distribution and the nematic order parameter in the limit of weak segregation are presented in Fig. 4. It is interesting to note that the nematic order parameter varies periodically following the periodicity of the lamellae phase, and possesses opposite signs in different blocks. One can readily see that NPs have a strong tendency to be localized in the regions with maximum concentration of monomers $A$ which interact stronger with them.

In the important limit when the NP-monomer interactions are short-ranged compared to the lamella block

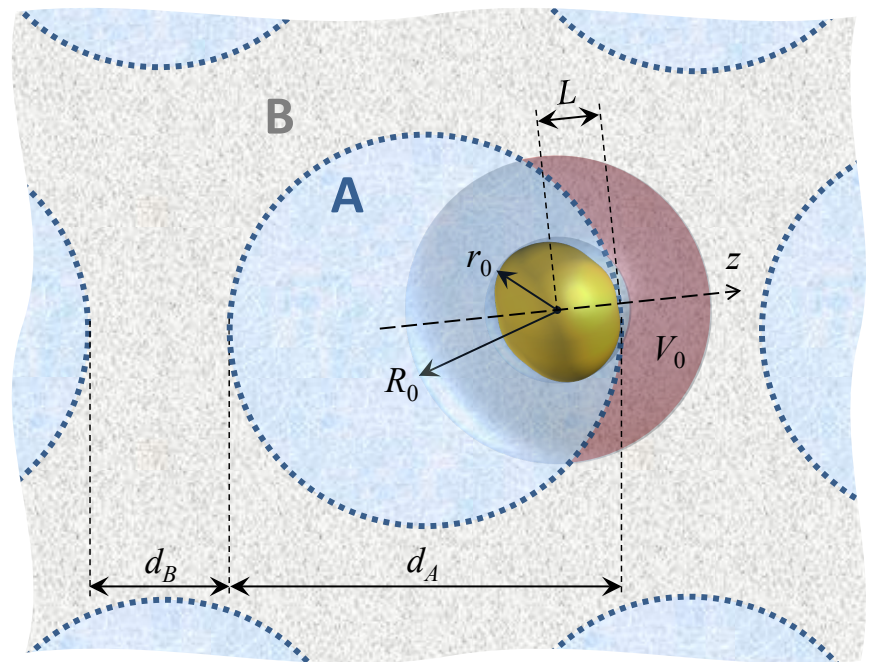

Fig. 5. NP embedded in a hexagonal diblock copolymer with strong segregation.

thickness, one can assume $q r \ll 1$ and then the folowing equation is obtained:

$$
\Delta J_{q} \approx 4 \pi \int \Delta J(r) r^{2} d r
$$

i. e., as independent of $q$, and hence

$$
\Delta I_{q} \approx-\frac{2 \pi}{5} q^{2} \int \Delta I(r) r^{4} d r
$$

The latter parameter induces the NP orientational ordering and, apparently, it can be estimated as $\left(r_{0} / d\right)^{2}$ times smaller than the parameter $\Delta J_{q}$ which is responsible for the NP positional segregation between different blocks.

\section{Hexagonal phase. Strong segregation}

Consider NPs embedded into a hexagonal diblock copolymer with strong segregation. We start with the case of NP inside the cylindrical A-block at the distance $L$ from the block boundary as shown in Fig. 5. We assume again that NP interacts with the monomers inside the spherical volume limited by the outer and inner radii $R_{0}$ and $r_{0}$ respectively. Similarly to Eq. (6), the mean-field potential can then be expressed as:

$$
\begin{array}{r}
U_{M F}=\rho_{0 A} \int_{r_{0}<r<R_{0}} d V\left[J_{A}+I_{A} P_{2}(\mathbf{a} \cdot \mathbf{u})\right] r^{-6} \\
+\int_{V_{0}} d V\left[\Delta J+\Delta I P_{2}(\mathbf{a} \cdot \mathbf{u})\right] r^{-6}
\end{array}
$$

where the first integral yields a potential that acts on the particle when its interaction sphere is completely inside the block A:

$$
U_{M F}^{(h 0)}(\mathbf{a}, L)=\frac{2 \pi}{3} \rho_{0 A} J_{A}\left(r_{0}^{-3}-R_{0}^{-3}\right) .
$$


This part of the potential is similar to Eq. (7) and does not depend on the NP location and orientation.

The second integral is taken over the volume confined between the interaction sphere surfaces and the cylindrical A-block surface, as highlighted in red in Fig. 5. The integration limits here can be determined by considering the intersection of the spherical and cylindrical surfaces. Let us use a local spherical coordinate system with the origin at the NP center $\mathbf{r}$ and the polar $z$-axis pointing towards the cylindrical A-block boundary (see Fig. 5).

The boundary of the A-block of the radius $d_{A} / 2$ in this coordinate system is described by the equation:

$$
\left(r \cos \theta+d_{A} / 2-L\right)^{2}+r^{2} \sin ^{2} \theta \cos ^{2} \phi=d_{A}^{2} / 4
$$

where the azimuthal angle $\phi$ is measured from the plane of the plot in Fig. 5. Altogether, the volume $V_{0}$ is confined between the surfaces $r=r_{0}, r=R_{0}$ and the cylinder described by Eq. (20).

Inside $V_{0}$, the radius is limited by $\max \left(r_{0}, L\right)<r<$ $R_{0}$, the azimuthal angle $0<\phi<2 \pi$, and the polar angle is $0<\theta<\theta_{m}(r, \phi)$ with the upper limit achieved on the cylinder surface (20). Solving the latter with respect to $\theta$ yields:

$$
\begin{aligned}
& \cos \theta_{m}(r, \phi)=\frac{d_{A}-2 L}{2 r \sin ^{2} \phi} \times \\
& {\left[\sqrt{1+4 \sin ^{2} \phi \frac{\left(d_{A}-L\right) L-r^{2} \cos ^{2} \phi}{\left(d_{A}-2 L\right)^{2}}}-1\right] .}
\end{aligned}
$$

Therefore, the second term in Eq. (18) can be expressed as

$$
\begin{gathered}
U_{M F}^{(h 1)}=\int_{\max \left(r_{0}, L\right)}^{R_{0}} r^{-4} d r \int_{0}^{2 \pi} d \phi \int_{0}^{\theta_{m}(r, \phi)} \sin \theta d \theta \times \\
{\left[\Delta J+\Delta I P_{2}(\mathbf{a} \cdot \mathbf{u})\right]=} \\
=\left(\Delta J-\frac{\Delta I}{2}\right) \int_{\max \left(r_{0}, L\right)}^{R_{0}} r^{-4} d r \int_{0}^{2 \pi} d \phi\left(1-\cos \theta_{m}\right)+ \\
\frac{\Delta I}{2} \int_{\max \left(r_{0}, L\right)}^{R_{0}} r^{-4} d r \int_{0}^{2 \pi} d \phi \times \\
{\left[P_{2}(\mathbf{a} \cdot \mathbf{k}) \cos \theta_{m} \sin ^{2} \theta_{m}+1-\cos \theta_{m}+\right.} \\
\left.\left(2+\cos \theta_{m}\right)\left(1-\cos \theta_{m}\right)^{2} \frac{1}{2}\left[(\mathbf{a} \cdot \mathbf{x})^{2}-(\mathbf{a} \cdot \mathbf{y})^{2}\right] \cos 2 \phi\right],
\end{gathered}
$$

where we have taken into account that $\theta_{m}(r, \phi)$ is an even function of $\phi$. Apparently, the next integration steps are to be taken numerically.

The expressions for the mean-field potential acting on NP centered outside the cylindrical block A are very similar if one introduces negative $L$ in this case. One has to account only for the fact that for $r<-L$ the maximum polar angle is $\theta_{m}=\pi$.

We have obtained the NP density and orientational order parameter profiles by a numerical integration of Eq. (4). A number of characteristic profiles are shown in Fig. 6.
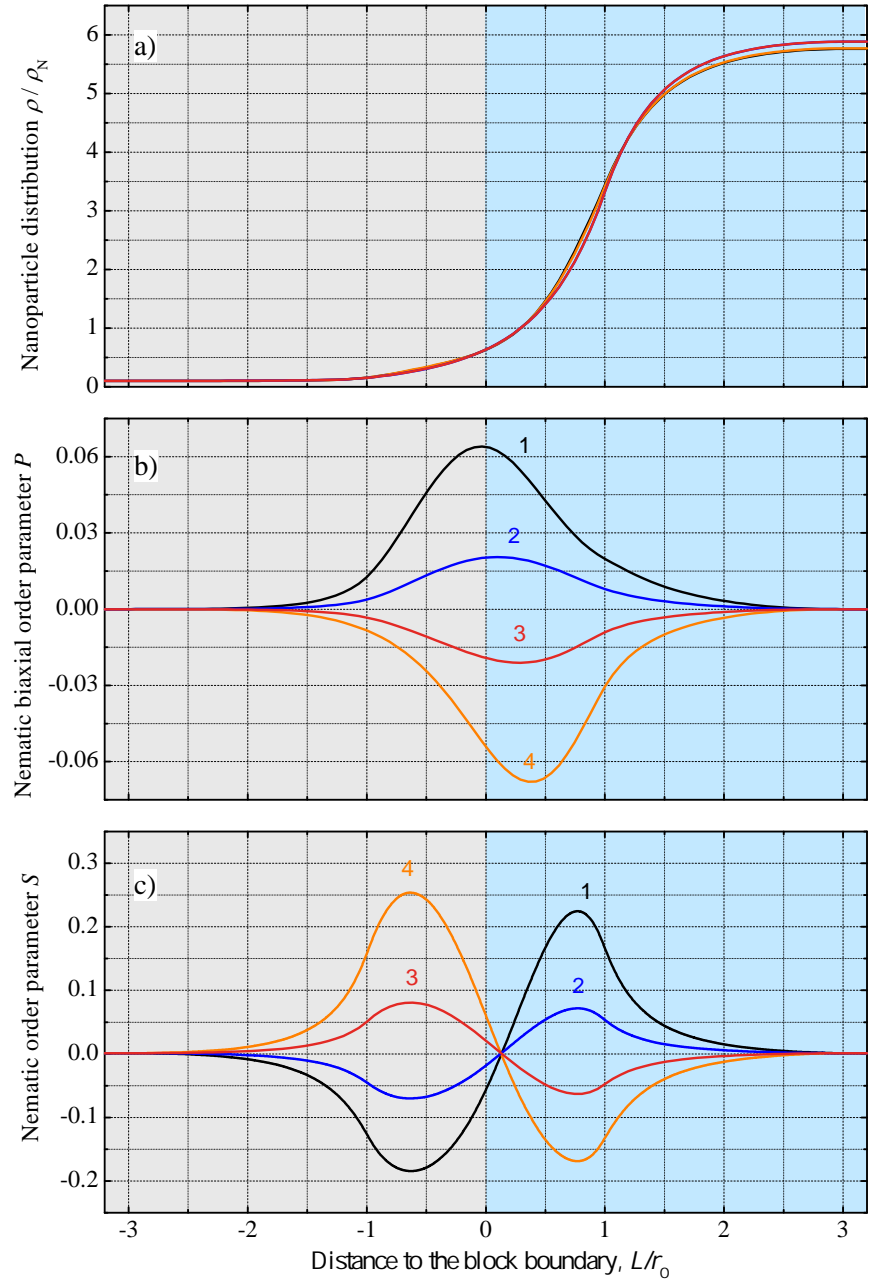

Fig. 6. NP local density, biaxial nematic and uniaxial nematic order parameters inside a hexagonal diblock copolymer with strong segregation. The block thickness parameters are is $d_{A}=d_{B}=7 r_{0}$, the interaction radius $R_{0}=3 r_{0}$, the isotropic interaction constant $\Delta J=k_{B} T$ and the anisotropic constant $\Delta I=-3 k_{B} T,-k_{B} T, k_{B} T, 3 k_{B} T$ for the lines from 1 to 4 respectively.

One notes that the point symmetry of the hexagonal phase in the vicinity of the cylindrical surface is biaxial due to the surface curvature. As a result, there exists a weak biaxial ordering of uniaxial NPs in this region. The biaxial nematic order parameter $P=\left\langle(\mathbf{a} \cdot \mathbf{x})^{2}-(\mathbf{a} \cdot \mathbf{y})^{2}\right\rangle$ profiles are also presented in Fig. 6. Note that in contrast to the uniaxial nematic order parameter, the biaxial order parameter possesses the same sign in both blocks and reaches its maximum exactly at the boundary.

The role of the cylindrical block boundary curvature is illustrated in Fig. 7 where the positional distribution of NPs and their uniaxial and biaxial order parameter profiles are presented in the vicinity of the cylindrical boundary for different values of its curvature. For comparison, we also present the corresponding profiles calculated near a flat boundary in a lamellae phase using the same interaction parameters. One can readily see that the curvature 

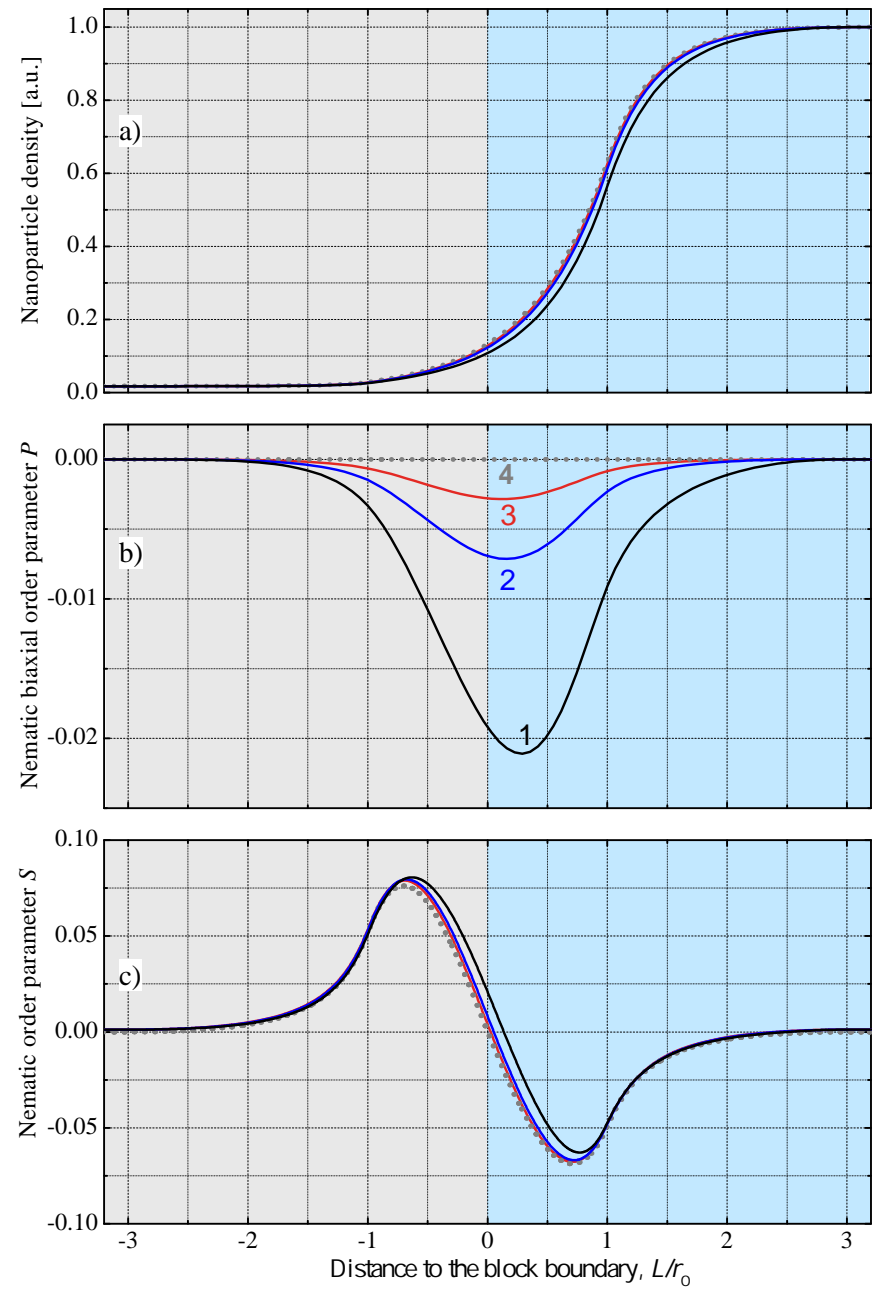

Fig. 7. The role of block boundary curvature: NP local density (a), biaxial nematic (b) and uniaxial nematic (c) order parameters inside a hexagonal diblock copolymer with the block diameter varying as $d_{A}=d_{B}=7 r_{0}, 20 r_{0}, 50 r_{0}$ for the lines from 1 to 3 respectively. The interaction radius $R_{0}=3 r_{0}$, the interaction constants are $\Delta J=k_{B} T$ and $\Delta I=k_{B} T$. The dotted line 4 is calculated for comparison for the lamellae copolymer with the same parameters.

only slightly affects the spatial distribution of NPs and the uniaxial nematic ordering. The biaxial nematic ordering, however, is determined by the curvature and strongly depends on it. Note also that for the chosen parameters the NP density near the boundary is rather low and only a small fraction of NPs is orientationally ordered.

\section{Discussion}

In this paper we have considered spatial distribution and orientational ordering of anisotropic NPs embedded into the lamellae and hexagonal phases of a block copolymer. NP concentration profiles and the nematic order parameter profiles of anisotropic NPs have been calculated in the lamellae and hexagonal phases in the limit of strong segregation taking into account both isotropic and anisotropic interactions between NPs and monomers. One notes that explicit analytical expression for the distribution of NPs in the lamellae phase have been derived. The translational and orientational distributions of NPs have also been calculated analytically in the lamellae phase in the opposite limit of weak segregation.

The role of isotropic interaction appears to be rather straightforward. If the isotropic interaction between NPs and monomers is sufficiently large, NPs are mainly located inside the block with the strongest interaction. A small fraction of particles also resides in the boundary region between the blocks. In contrast, the anisotropic interaction between NPs and monomers (which is not taken into consideration in the existing molecular theory of polymer nanocomposites) is responsible for a number of interesting phenomena in the boundary region.

If NP is located inside a particular block far enough from its boundaries, the anisotropic interaction is averaged out and the orientational order parameter of anisotropic NPs vanishes. At the same time, if NP is sufficiently close to a boundary between two blocks, it may interact simultaneously with the monomers of both blocks. The corresponding anisotropic interaction constants are generally different, and this asymmetry induces some orientational order of NPs in the boundary region. Moreover, the sign of the nematic order parameter is different in the two blocks, that is the rod-like NPs are expected to align parallel to the interface in one block and perpendicular to the interface in the other. Taking into account that the density of NPs is different in different blocks in the boundary region, one concludes that there is a nonzero average nematic order in the block copolymer. The corresponding average orientational order parameter is small but it may be sufficient to align the polymer by external electric or magnetic field if the NP anisotropy is sufficiently large. These theoretical results are qualitatively confirmed by the experiment. For example, functionalized gold NRs are aligned parallel or perpendicular to the surface of the cylindrical domain in the hexagonal phase [41-43]. Similar gold NRs have also been found to align parallel to the flat block boundary in the lamellae phase [40]. One notes that the theoretical profiles describe the average distribution and orientation of NPs. In real systems, the particles are sometimes rather large and their concentration is low. In this case, the distribution is essentially discrete and individual NPs may be aligned parallel or perpendicular to the interface depending on their location in a particular block.

At present, there are few experimental data on the orientational ordering of NPs in block copolymers [40-43], and all of these data have been obtained using rather large NPs with dimensions comparable to the size of a block. It would be interesting to decrease the size of anisotropic NPs and increase their molar fraction. In this way, it will be possible to verify the qualitative prediction of the theory which indicates that NPs are expected to align perpendicular to the boundary (between the two blocks) in one block and parallel to the boundary in another block.

There is an interesting special case when the isotropic interaction between NPs and monomers of blocks A and B 
is approximately the same while their anisotropic interaction is different. Then the concentration of NPs is nearly the same in both blocks away from the boundaries, while the NP concentration in the boundary region is higher than in the bulk. Anisotropic NPs are also orientationally ordered in the boundary region.

In the hexagonal phase, the NP spatial distribution and their nematic order parameter profiles are qualitatively similar to those in the lamellae phase. In addition, the curvature of the cylindrical surface induces a small biaxiality in the orientational distribution of uniaxial NPs in the interfacial region, although the corresponding biaxial order parameter stays rather small. Note that the biaxial order parameter has been calculated above in a local coordinate system. Biaxial order will vanish identically after averaging over the whole uniaxial diblock periodic structure. The same is valid for the uniaxial nematic order parameter of NPs in a body-centered cubic diblock copolymer with spherical blocks. The local values of the order parameter is nonzero but on average there is no orientational order in this case as the system does not have a preferred direction on average. In the case of spherical blocks, the local biaxial order occurs only if the primary axis of a NP is aligned on average parallel to the spherical boundary because in this geometry the two local directions perpendicular to the preferred direction of ordering are not equivalent. In contrast, if the preferred direction of the NP axis is perpendicular to the spherical block boundary, the two other orthogonal directions are equivalent and hence there is no local biaxial order.

One notes also that the inhomogeneous distribution of NPs in the lamellae and hexagonal phases as well as the orientational ordering of anisotropic NPs in the interfacial region between the blocks generally contributes both to the bulk and to the interfacial free energy of these phases. As a result, NPs may affect the relative stability of these phases and shift the parameters of the corresponding phase transitions. A molecular theory, which takes these effects into account will be developed in our future works.

\section{Acknowledgements}

MAO is grateful to the Russian Science Foundation (project 16-13-10280).

\section{Author contribution statement}

The authors contributed equally to the paper.

\section{References}

1. M. Alexandre, P. Dubois, Mater. Sci. Eng. Rev. 28, (2000) 1.

2. A. C. Balazs, Curr. Opin. Colloid Interface Sci. 4, (2000) 443.

3. E. P. Giannelis, Appl. Organomet. Chem. 12, (1998) 675.
4. P. P. Soo, B. Y. Huang, Y. I. Jang, Y. M. Chiang, D. R. Sadoway, A. M. Mayes, J. Electrochem. Soc. 146, (1999) 32. 5. A. C. Balazs, T. Emrick, T. P. Russell, Science 314 (2006) 1107.

6. R. Krishnamoorti, R. A. Vaia, J. Polym. Sci., Part B: Polym. Phys. 45, (2007) 3252.

7. A. J. Crosby and J. Y. Lee, Polym. Rev. 47, (2007) 217.

8. D. Zhao, J. Feng, Q. Huo, N. Melosh, G. H. Fredrickson, B. F. Chmelka, G. D. Stucky, Science 279, (1998) 548.

9. V. Z. H. Chan, J. Hoffman, V. Y. Lee, H. Iatrou, A. Avgeropoulos, N. Hadjichristidis, R. D. Miller, E. L. Thomas, Science 286, (1999) 1716.

10. Y. Lin, A. Boker, J. B. He, K. Sill, H. Q. Xiang, C. Abetz, X. F. Li, J. Wang, T. Emrick, S. Long, Q. Wang, A. C. Balazs, T. P. Russell, Nature '434, (2005) 55.

11. J. J. Chiu, B. J. Kim, E. J. Kramer, D. J. Pine, J. Am. Chem. Soc. 127, (2005) 5036.

12. S. Park, J. Wang, B. Kim, T. P. Russell, Nano Lett. 8, (2008) 1667.

13. H. Kang, F. A. Detcheverry, A. N. Mangham, M. P. Stoykovich, K.Ch. Daoulas, R. J. Hamers, M. Muller, J. J. de Pablo, P. F. Nealey, Phys. ReV. Lett. 100, (2008) 148303. 14. R. B. Thompson, V. V. Ginzburg, M. W. Matsen, A. C. Balazs, Science 292, (2001) 2469.

15. M. R. Bockstaller, Y. Lapetnikov, S. Margel, E. L. Thomas, Journal of the American Chemical Society 125, (2003) 5276.

16. M. R. Bockstaller, R. A. Mickiewicz, E. L. Thomas, Adv. Mater. 17, (2005) 1331.

17. M. J. A. Hore, R. J. Composto, ACS Nano 4, (2010) 6941. 18. A. L. Frischknecht, M. J. Hore, J. Ford, R. J. Composto, Macromolecules 46, (2013) 2856.

19. M. J. Hore, R. J. Composto, Macromolecules 47, (2014) 875.

20. Q.-Y. Tang, Y.-Q. Ma, J. Phys. Chem. B 113, (2009) 10117.

21. B. Rasin, H. Chao, G. Jiang, D. Wang, R. A. Riggleman, R. J. Composto, Soft Matter 12, (2016) 2177.

22. Z. Shou, G. A. Buxton, A. C. Balazs, Composite Interfaces 10, (2003) 343.

23. S. Sides, B. Kim, E. Kramer, G. Fredrickson, Phys. Rev. Lett. 96, (2006) 250601.

24. V. Ganesan, A. Jayaraman, Soft Matter 10, (2014) 13.

25. H. Qi, B. Kinkead, T. Hegmann, Proc. SPIE. 6911, (2008) 691106.

26. H. Qi, T. Hegmann, J. Mater. Chem. 16, (2006) 4197.

27. Y. Shiraishi, N. Toshima, H. Maeds, K.and Yoshikawa, J. Xu, S. Kobayashi, Appl. Phys. Lett. 81, (2002) 2845.

28. S. Kobayashi, N. Toshima, Information Display 23, (2007) 26.

29. H. Yoshida, K. Kawamoto, H. Kubo, A. Tsuda, T.and Fujii, S. Kuwabata, M. Ozaki, Adv. Mater. 22, (2010) 622.

30. S. Kaur, S. P. Singh, A. M. Biradar, A. Choudhary, K. Sreeniva, Appl. Phys. Lett. 91, (2007) 023120.

31. A. Kumar, J. Prakash, A. M. Mehta, D. S. Biradar, W. Haase, Appl. Phys. Lett. 95, (2009) 023117.

32. Y. Reznikov, O. Buchnev, O. Tereshchenko, V. Reshetnyak, A. Glushchenko, J. West, Appl. Phys. Lett. 82, (2003) 1917.

33. S. Wiersma, Nature Physics 4, (2008) 359.

34. V. Gdovinova, N. Tomasovicova, N. Eber, T. Toth-Katona, V. Zavisova, M. Timko, P. Kopcansky, Liq. Cryst. 41, (2014) 1773 . 
35. M. V. Gorkunov, M. A. Osipov, Soft Matt. 7, (2011) 4348.

36. L. M. Lopatina, J. V. Selinger, Phys. Rev. E. 84, (2011) 041703.

37. M. V. Gorkunov, G. A. Shandryuk, A. M. Shatalova, I. Y. Kutergina, A. S. Merekalov, Y. V. Kudryavtsev, R. V. Talroze, M. A. Osipov, Soft Matt. 9, (2013) 3578.

38. M. A. Osipov, M. V. Gorkunov, ChemPhysChem 15, (2014) 1496.

39. M. A. Osipov, M. V. Gorkunov, Phys. Rev. E. 92, (2015) 032501.

40. R. D. Deshmukh, Y. Liu, R. J. Composto, NanoLett. 7, (2007) 3662.

41. E. Ploshnik, A. Salant, U. Banin, R. Shenhar, Adv. Mater. 22, (2010) 2774.

42. E. Ploshnik, A. Salant, U. Banin, R. Shenhar, Phys. Chem. Chem. Phys. 12, (2010) 11885.

43. K. Thorkelsson, A. J. Mastroianni, P. Ercius, and T. Xu, NanoLett. 12, (2012) 498.

44. B. W. Van der Meer and G. Vertogen in G. R. Luckhurst and G. W. Gray (eds.) Molecular Physics of Liquid Crystals (Academic Press, New York 1979).

45. M. A. Osipov, Molecular theories of liquid crystals, in J. W. Goodby, P. J. Collins, T. Kato, C. Tschierske, H. F. Gleeson, P. Raynes (eds.) Handbook of Liquid Crystals: Vol.1. Fundamentals of Liquid Crystals (Wiley-VCH, Weinheim 2014), 115-168.

46. M. V. Gorkunov, M. A. Osipov, J. P. F. Lagerwall, and F. Giesselmann, Phys. Rev. E 76, (2007) 051706.

47. L. Leibler, Macromolecules 13, (1980) 1602. 